Reducing avoidable admissions in rural community palliative care: a pilot study of care coordination by General Practice registrars

Thea F. van de Mortel (50\%)*

A/Deputy Head of School (Learning \& Teaching),

School of Nursing \& Midwifery, Griffith University

Parklands Drive, Southport, QLD, 4222

Ph: 0755527855 Email: t.vandemortel@griffith.edu.au

Kenneth Marr (15\%)

A/Director of Palliative Care, Northern NSW Local Health District

Liz Burmeister (10\%)

Nurse Researcher, Princess Alexandra Hospital

Hilton Koppe (5\%)

Senior Medical Educator, North Coast GP Training

Christine Ahern (5\%)

Director of Training, North Coast GP Training

Robert Walsh (5\%)

General Practitioner, Tintenbar Medical Centre

Susan Tyler-Freer (5\%)

GP registrar, North Coast GP Training

Dan Ewald (5\%)

North Coast Primary Health Network

Disclosure statement

The registrar service was funded and collaboratively administered by the Northern NSW Local Health District and the North Coast NSW Medicare Local (now North Coast Primary Health Network). Registrar support was provided by North Coast GP Training. Griffith University conducted the evaluation.

Acknowledgements

We gratefully acknowledge the RACGP Foundation and the Independent Practitioner Network Ltd for their support of the evaluation component of this project. We would also like to acknowledge the ongoing support of Vahid Saberi (North Coast Primary Health Network), Vicki Rose (Northern NSW Local Health District), John Langill (North Coast GP Training), the team of palliative care and community nurses, and local General Practitioners.

Conflicts of interest Nil. 


\section{Reducing avoidable admissions in rural community palliative care: a pilot study of care coordination by General Practice registrars}

Abstract

Objective: To investigate the feasibility of using a General Practice registrar (GPR) to coordinate rural palliative care services by examining the preliminary outcomes of service implementation.

Design: A quasi-experimental design was used. Intervention group participants received the GPR service, which involved liaison between the patient, family, General Practitioner, specialist palliative care team and community nurses. Specified risk assessment, care planning and continuity of care were provided. Patients in the comparison group received the standard service.

Setting: Rural community palliative care.

Participants: One hundred and ninety-one rural community palliative care patients (99 intervention and 92 control).

Main outcome measures: Hospital admissions per 100 patient days, bed days per 100 patient days and proportion of deaths at home.

Results: Patients receiving standard care were twice as likely to spend $\geq 8$ bed-days in hospital (OR $2.09(95 \% \mathrm{Cl} 1.10-3.97) ; \mathrm{p}=0.02)$ and were more likely to have $\geq$ two admissions to hospital (OR 3.37 (95\% Cl 1.83-6.21); $p<0.001)$, per 100 patient-days than the intervention group after adjusting for diagnosis group (cancer or not) and residence in residential aged care. Controls were significantly less likely to die at home than the intervention group (OR $0.41(95 \% \mathrm{Cl} 0.20-0.86) ; \mathrm{p}=0.02)$.

Conclusion: This is a small proof of concept pilot study limited by lack of randomisation. The results demonstrate the feasibility of using a GPR to manage continuity of care for rural community palliative care patients. Further investigation via a randomised controlled trial is required.

\section{What is already known on this subject}

- Most palliative care patients prefer to die at home but the majority die in hospital.

- Avoidable admissions are caused by lack of after-hours community palliative care, fragmented care, and the variable palliative care expertise and availability of General Practitioners (GPs).

- There is insufficient information in the Australian rural setting on a model of community palliative care that addresses these issues.

\section{What this paper adds}

- The results of this proof of concept pilot study demonstrated the feasibility of using a GP Registrar to manage continuity of care for rural community palliative care patients.

- This model has the potential to smooth out variations in GP availability and expertise through improved communication between the GPs, specialists, nurses and the patient and their family, which may provide superior continuity of care from multiple GPs.

- Further research with a larger randomised sample is required to confirm these findings. 
Introduction

Palliative care separations have increased by $51 \%$ over a decade, ${ }^{1}$ and demand is projected to rise $4.6 \%$ annually. ${ }^{2}$ Community palliative care is $50 \%-300 \%$ less expensive than hospital-based care, yet whilst $74 \%$ of Australians wish to die at home only $16 \%$ actually do. ${ }^{2}$ In 2009-2010 56,000 palliative care separations occurred in Australian hospitals, involving 663,000 bed-days, and a length of stay four times longer than average. ${ }^{1}$ Unplanned admissions occur due to lack of after-hours care, inadequate symptom management and poorly coordinated care..$^{2-4}$ Care can be fragmented when delivered by multiple health professionals, ${ }^{3,5}$ and often happens following crises rather than as a planned trajectory, particularly when patients have not completed an Advance Care Directive (ACD). ${ }^{2}$ Community palliative care delivery is particularly problematic for rural communities due to infrastructure/resource constraints, and sparse population distribution. ${ }^{5}$

A systematic review concluded that policy should focus on intensifying home care. ${ }^{6}$ General Practitioners (GPs) should play a central role as palliative care delivery by GPs reduces hospitalisations ${ }^{7}$ and increases home deaths. ${ }^{8-10}$ Barriers to involvement include unwillingness to provide after-hours care and lack of experience in palliative medicine, ${ }^{8,11-13}$ as Australian GPs only see 5-8 terminally ill patients annually. ${ }^{14-16}$ Rural GPs may also find it difficult to access specialist advice. ${ }^{17}$

The National Health and Hospitals Reform Commission recommends 'integrating multi-disciplinary primary health care services...better coordination and continuity of care...[and] strengthening consumer...empowerment' ${ }^{2}$ High quality end-of-life care involves managing multiple co-morbidities, symptom control, and 'psychological, social, loss/grief, practical and end-of-life preparation'. ${ }^{18}$ Strategies include:

- Empowering consumers through ACD uptake, ${ }^{2}$

- improving communication between GPs and specialist palliative care teams to improve care coordination ${ }^{15}$ and provide opportunities for GPs to strengthen palliative medicine skills, ${ }^{5,16}$

- providing after-hours and planned care based on regular risk assessments using end-of-life tools to maintain continuity of care and reduce admissions. ${ }^{6}$

This study examined the feasibility of using a GP registrar (GPR) to facilitate communication between palliative care specialists, consumers and GPs, and provide risk assessment, care planning and continuity of care.

Methods

A quasi-experimental design utilised an intervention and comparison group. In order to manage the logistical constraints of service delivery over a large rural area, intervention group participants were drawn from community nursing area 2 (CNA2), and the comparison group from two others. Participants were referred by general practices and specialists to the palliative care service. Adults $\geq$ 18 years with a terminal illness were eligible to participate. Potential participants (or Person Responsible if unfit to consent) were given an Information Sheet on study aims and completed a consent form to receive the registrar service. The data were de-identified to protect patient confidentiality.

Ethics approval was obtained from the North Coast NSW Human Research Ethics Committee (LNR060). Both groups received standard care (Box 1), but the intervention group had their care 
coordinated by the registrar after consultation with the patients' GP, under the supervision of the Director of Palliative Care. The same two GPRs provided care throughout the study period. They conducted the initial patient assessment, and case conferenced with the medical and nursing teams and the family to develop the care plan. They also conducted 3-month follow-up (for stable patients) or re-assessed and updated the plan if the patient deteriorated. This service was initially available during business hours but was extended to after-hours as funding became available. In comparison CNAs patient assessment, planning and care coordination was conducted by palliative care nurses.

Age, gender, marital status, number of carers, diagnosis, and postcode were recorded on enrolment. Resource Utilisation Group-Activities of Daily Living (RUG-ADL) and Australian-Modified Karnofsky Performance Scale (AKPS) scores guided functional assessment. A decrease in AKPS and increase in RUG-ADL scores indicate a decline in functional performance and increased dependency. ${ }^{19}$ Other data routinely collected by clinicians using a data collection sheet and de-identified at death included:

- Date and source of referral,

- Date and place of death,

- Preferred place of care and death,

- Completion of an ACD,

- Dates of hospital admissions, reason for admission and number of bed-days per admission.

Missing or anomalous data were checked against unit records. Outcome measures included the proportion of home deaths and proportion with an ACD, and number of hospital admissions and bed-days per 100 patient-days.

Data were analysed using Stata14. ${ }^{20}$ Descriptive statistics were calculated and differences between groups were tested using chi-squared or exact tests for categorical data and Wilcoxon rank-sum tests for continuous variables. Bed-days and admissions were calculated using the number of days or occasions admitted to a hospital, respectively, between recruitment and death, corrected for the number of days enrolled in the study and expressed per 100 patient-days. Because of the nonnormal distribution, outcomes of bed-days and admissions were categorized into clinically and statistically meaningful groups.

Logistic Regression was used to determine associations between factors and ACD completion, home death, admissions $(\geq 2,<2)$ and bed-days $(\geq 8,<8)$ per 100 patient days. Independent factors investigated included gender, age $(<75, \geq 75$ years) diagnosis (cancer or other), marital status (no partner, partner), AKPS ( $\leq 40,50,60$ or $\geq 70)$, and RUG-ADL scores (1-5, 6-10 or $\geq 11)$. For the outcomes, statistically significant predictor variables (at $p=0.10$ ) were identified using bivariate analyses. These were then included in the multivariate analyses. Multivariate logistic analyses were conducted for each outcome, which included the treatment group in all models as an independent factor to estimate the effect of the intervention on each outcome adjusting for significant clinical and statistical factors. Models were assessed using likelihood ratio tests and Wald statistics. Results for these models are reported using odds ratios (ORs) and 95\% confidence intervals (Cls) for each factor. 
The sample size analysis estimated that 50 patients in each group would be required to show a $30 \%$ difference in the proportion of patients who died at home between groups, with a power of $80 \%$ and an alpha level of 0.05 .

Results

Ninety-nine intervention and 92 comparison patients participated over two years. The intervention group was more dependent at recruitment (Table 1), more were referred by a GP (72\% vs $49 \%$; $p$ $<0.001$ ), fewer had cancer ( $71 \%$ vs $84 \% ; p=0.02$ ), and more completed an ACD ( $83 \%$ vs $22 \%$; $<0.001$ ). Residential Aged Care (RAC) was the preferred place of care for $31 \%$ of intervention group participants versus $2 \%$ of comparisons.

Predictor variables are shown in Table 2. A multivariate model that adjusted for diagnosis and RAC residence found the comparison group were twice as likely to have zeight bed-days than the intervention group (OR $2.09(95 \% \mathrm{Cl} 1.10-3.97) ; \mathrm{p}=0.024)$ per 100 days (Table 3). After adjusting for age and RAC residence, multivariate analysis determined that the comparison group were more likely to have $\geq$ two admissions than the intervention group (OR $2.67(95 \% \mathrm{Cl} 1.39-5.11) ; p<0.003$ ) per 100 days.

Given $88 \%$ of RAC patients died in RAC, and more intervention group patients were residents on enrolment, when examining home deaths, RAC patients were excluded from the analysis. The comparison group were significantly less likely to die at home than the intervention group (OR 0.41(95\% Cl 0.20-0.86); $\mathrm{p}=0.02)$ (Table 1$)$.

Discussion

These results demonstrate the feasibility of using a GPR to manage continuity of care for rural community palliative care patients. The percentage of intervention group patients dying at home overall (30\%) compared favourably with those (16-19\%) reported in Australian palliative care populations. ${ }^{21}$ While more comparison patients had cancer, they were younger, more likely to be married and less care dependent at recruitment than the intervention group. Younger age and married status are associated with higher rates of home death. ${ }^{21,22}$ Adequate carer support is also a key component of successful home death, although lower functional status has been associated with a higher proportion of home deaths. ${ }^{6}$ Despite this the comparison group were more likely to be hospitalised, and had more bed days.

The GP registrar care coordination addressed a key barrier to optimal community care of the dying: insufficient medical support, ${ }^{4}$ which can be problematic in rural communities. Consumer engagement through ACD completion was also addressed, with a significant improvement in completion rates. Forward planning using instruments to measure functional status, combined with as needed home visits to pre-empt crises provided a solid platform on which to deliver quality community palliative care. Better care coordination has been demonstrated to improve home death rates in the international setting. ${ }^{18}$

Future studies will require a more reliable indicator of socio-economic status as estimation based on socio-economic disadvantage by postcode, ${ }^{23}$ is not ideal. ${ }^{24}$ Additionally, as care was delivered in CNAs and GP practices were used to recruit participants, patients were not randomised to group, 
which is likely to introduce confounders. Also, a higher proportion of intervention group patients were referred by GPs, which may have biased towards a better fit of the program to patients' needs.

\section{Conclusions}

Expertise varies greatly between GPs. Medical speciality registrars servicing multiple practices in a defined area may have the potential to smooth out these variations. This small proof of concept pilot demonstrated the feasibility of using a GPR to manage continuity of care for rural community palliative care patients. Further research is required to test the potential benefits. Potential cost efficiencies and stakeholder's views on quality of life impacts should also be examined. 


\section{References}

1 Australian Institute of Health and Welfare. Palliative care services in Australia 2012. Canberra:AlHW, 2012.

2 Palliative Care NSW. Palliative Care in NSW Policy Statement. 2012. Available from: https://palliativecarensw.org.au/site/wp-content/uploads/2012/05/PCNSW-Policy-Statement-FINAL1.pdf 3 Aubin M, Giguere A, Martin M, et al. Interventions to improve continuity of care in the follow-up of patients with cancer. Cochrane Library 2012;2012(7).

4 Hardy J, Maresco-Pennisi D, Gilshenan K, Yates P. Barriers to the best care dying in Queensland, Australia. Journal of Palliative Medicine 2008;11(10):1325-9.

5 Evans R, Stone S, Elwyn G. Organizing palliative care for rural populations: a systematic review of the evidence. Family Practice 2003;20(3):304-10.

6 Gomes B, Higginson IJ. Factors influencing death at home in terminally ill patients with cancer: systematic review. British Medical Journal 2006;332 515-21.

7 Van den Block L, Deschepper R, Drieskens K, et al. Hospitalisations at the end of life: using a sentinel surveillance network to study hospital use and associated patient, disease and healthcare factors. BMC Health Services Research 2007;7:69.

8 Rhee JJ, Zwar N, Vagholkar S, Dennis S, Broadbent AM, Mitchell G. Attitudes and barriers to involvement in palliative care by Australian urban general practitioners. Journal of Palliative Medicine 2008;11(7):980-5. 9 Neergaard MA, Vedsed P, Olesen F, Sokolowski I, Jensen AB, Sondergaard J. Associations between home death and GP involvement in palliative cancer care. British Journal of General Practice 2009 59:671-7. 10 Aabom B, Kragstrup J, Vondeling H, Bakketeig LS, Stovring H. Does persistent involvement by the GP improve palliative care at home for end-stage cancer patients? Palliative Medicine 2006;20:507-12. 11 Groot MM, Vernooij-Dassen MJFJ, Crul BJP, Grol RPTM. General practitioners and palliative care: perceived tasks and barriers in daily practice. Palliative Medicine 2005;19:111-8.

12 Shipman C, Gysels M, White $P$, et al. Improving generalist end of life care: national consultation with practitioners, commissioners, and service user groups. British Medical Journal 2008;337:a1720.

13 Yuen KJ, Behrndt M, Jacklyn C, Mitchell G. Palliative care at home: general practitioners working with palliative care teams. Medical Journal of Australia 2003;179:S38-S40.

14 Pereira GJ. Palliative care in the hinterlands: A description of existing services and doctors' attitudes. Australian Journal of Rural Health 2005;13:343-7.

15 Mitchell G. How well do general practitioners deliver palliative care? Palliative Medicine 2002;16: 45764.

16 Mitchell GK, Reymond EJ, McGrath PM. Palliative care: promoting general practice participation. Medical Journal of Australia 2004;180(5):207-8.

17 Smyth D, Farnell A, Dutu G, Lillis S, Lawrenson R. Palliative care provision by rural general practitioners in NZ. Journal of Palliative Medicine 2009; 13(3):247-50.

18 Howell D, Abernathy T, Cockerill R, Brazil K, Wagner F, Librach L. Predictors of home care expenditures and death at home for cancer patients in an integrated comprehensive palliative home care pilot program. Healthcare Policy 2011;6(3):e73-e92.

19 Clapman S, Holloway A. Palliative care outcomes collaboration: clinical manual. 2014. Available from: https://ahsri.uow.edu.au/content/groups/public/@web/@chsd/@pcoc/documents/doc/uow129133.pdf 20 Stata Corp. STATA. 13.0 ed. College Station, TX.

21 Howatt A, Veitch C, Cairns W. A retrospective review of place of death of palliative care patients in regional north Queensland. Palliative Medicine 2007;21:41-7.

22 Burge F, Lawson B, Johnson G. Trends in the place of death of cancer patients 1992-1997. Canadian Medical Association Journal 2003;168:265-70.

23 Australian Bureau of Statistics. 2033.0.55.001- Socioeconomic Indexes for Areas (SEIFA), Data Cube Only, 2011. 2013; Available from:

http://www.ausstats.abs.gov.au/Ausstats/subscriber.nsf/0/22CEDA8038AF7A0DCA257B3B00116E34/\$File/ 2033.0.55.001\%20seifa\%202011\%20technical\%20paper.pdf.

24 Ben-Schlomo Y, Smith GD. Commentary: Socioeconomic position should be measured accurately. British Medical Journal 1999;318:844-5. 
Box 1. Standard services.

\section{Palliative Services}

Initial assessment

Care plan teleconference with GP and palliative care team

Advance Care Plan creation

Three monthly monitoring visits

Acute symptom assessment/management plus specialist teleconference as required

End-of-Life Care Plan assessment (when AKPS $\leq 40$ )

\section{End-of-Life Services}

End-of-Life Care pathway initiation

Death certification

Bereavement follow-up 
Table 1. Patient characteristics.

\begin{tabular}{|c|c|c|c|c|}
\hline & $\begin{array}{c}\text { Total } \\
(n=191)\end{array}$ & $\begin{array}{c}\text { Intervention } \\
(n=99)\end{array}$ & $\begin{array}{l}\text { Control } \\
(n=92)\end{array}$ & P-value* \\
\hline \multicolumn{5}{|l|}{ Characteristics } \\
\hline \multicolumn{5}{|l|}{ Gender } \\
\hline Female & $82(43 \%)$ & $43(43 \%)$ & $39(42 \%)$ & \multirow{2}{*}{0.88} \\
\hline Male & $109(57 \%)$ & $56(56 \%)$ & $53(58 \%)$ & \\
\hline \multicolumn{5}{|l|}{ Age } \\
\hline$<75$ & $95(50 \%)$ & $43(43 \%)$ & $52(57 \%)$ & \multirow{2}{*}{0.07} \\
\hline$\geq 75$ & $96(50 \%)$ & $56(57 \%)$ & $40(43 \%)$ & \\
\hline \multicolumn{5}{|l|}{ Diagnosis } \\
\hline Cancer & $148(77 \%)$ & $70(71 \%)$ & $76(84 \%)$ & \multirow[t]{2}{*}{0.02} \\
\hline Other & $43(23 \%)$ & $29(29 \%)$ & $14(16 \%)$ & \\
\hline Missing & 2 & & 2 & \\
\hline \multicolumn{5}{|l|}{ Marital status } \\
\hline Single/widowed/divorced & $90(47 \%)$ & $50(51 \%)$ & $40(43 \%)$ & \multirow[t]{2}{*}{0.33} \\
\hline Married/de facto & $101(53 \%)$ & $49(49 \%)$ & $52(57 \%)$ & \\
\hline \multicolumn{5}{|l|}{ AKPS } \\
\hline$\leq 40$ & $57(30 \%)$ & $40(40 \%)$ & $17(18 \%)$ & \multirow{4}{*}{0.01} \\
\hline 50 & $42(22 \%)$ & $18(18 \%)$ & $24(26 \%)$ & \\
\hline 60 & $59(30 \%)$ & $27(26 \%)$ & $32(35 \%)$ & \\
\hline $70+$ & $33(17 \%)$ & $14(14 \%)$ & $19(21 \%)$ & \\
\hline \multicolumn{5}{|l|}{ RUG-ADL } \\
\hline $1-5$ & $82(43 \%)$ & $33(33 \%)$ & $49(53 \%)$ & \multirow{3}{*}{$<0.001$} \\
\hline $6-10$ & $64(34 \%)$ & $29(29 \%)$ & $35(38 \%)$ & \\
\hline $11+$ & $45(24 \%)$ & $37(37 \%)$ & $8(9 \%)$ & \\
\hline \multicolumn{5}{|c|}{ Referral and Preferences for care } \\
\hline Source Referral & & & & \multirow{5}{*}{0.001} \\
\hline General Practitioner & $113(61 \%)$ & $71(72 \%)$ & $42(49 \%)$ & \\
\hline Specialist & $70(38 \%)$ & $26(26 \%)$ & $44(51 \%)$ & \\
\hline Other & $2(1 \%)$ & $2(2 \%)$ & 0 & \\
\hline Unknown & 6 & 0 & 6 & \\
\hline \multicolumn{5}{|l|}{ Preferred place of care } \\
\hline Home & $130(71 \%)$ & $57(61 \%)$ & $73(83 \%)$ & \multirow[t]{4}{*}{$<0.001$} \\
\hline Hospital & $21(12 \%)$ & $8(9 \%)$ & $13(15 \%)$ & \\
\hline RAC & $31(1 \%)$ & $29(31 \%)$ & $2(2 \%)$ & \\
\hline Unknown & 9 & 5 & 4 & \\
\hline \multicolumn{5}{|l|}{ Preferred place of death } \\
\hline Home & $110(61 \%)$ & $52(55 \%)$ & $58(67 \%)$ & \multirow[t]{5}{*}{$<0.001$} \\
\hline Hospital & $31(17 \%)$ & $13(14 \%)$ & $18(21 \%)$ & \\
\hline Hospice & $9(5 \%)$ & $2(2 \%)$ & $7(8 \%)$ & \\
\hline RAC & $31(17 \%)$ & $27(29 \%)$ & $4(5 \%)$ & \\
\hline Unknown & 10 & 5 & 5 & \\
\hline \multicolumn{5}{|l|}{ Place of Death } \\
\hline Home & $47(27 \%)$ & $26(31 \%)$ & $21(24 \%)$ & \multirow[t]{4}{*}{$<0.001$} \\
\hline Hospital & $83(48 \%)$ & $28(33 \%)$ & $55(62 \%)$ & \\
\hline RAC & $44(25 \%)$ & $31(36 \%)$ & $13(15 \%)$ & \\
\hline Unknown $^{a}$ & 17 & 14 & 3 & \\
\hline \multicolumn{5}{|l|}{ ACD completed } \\
\hline No & $89(47 \%)$ & $17(17 \%)$ & $72(78 \%)$ & $<0.001$ \\
\hline Yes & $101(53 \%)$ & $81(83 \%)$ & $20(22 \%)$ & \\
\hline Unknown & 1 & 1 & 0 & \\
\hline
\end{tabular}

RAC= Residential Aged Care; ACD=Advance Care Directive; RUG-ADL=Resource Utilisation Group-Activities of Daily Living; AKPS=Australian-Modified Karnofsky Performance Scale *calculated using Pearson $\mathrm{Chi}^{2}$ and Fisher's Exact test.

aSome participants relocated and were consequently discharged from the service. 
Table 2. Association between patient factors and 1) bed-days ( $n=191), 2)$ admissions $(n=191), 3)$ death at home $(n=130)^{a}$ and 4$)$ advanced care directive completion $(n=190)$.

\begin{tabular}{|c|c|c|c|c|}
\hline & \multicolumn{4}{|c|}{ Odds ratio (95\% Confidence interval) } \\
\hline & $\geq 8$ Bed-days & $\geq 2$ Admissions & Home death & $\begin{array}{l}\text { Advance Care } \\
\text { Directive } \\
\text { completion }\end{array}$ \\
\hline \multicolumn{5}{|l|}{ Protocol } \\
\hline Intervention & 1 & 1 & 1 & 1 \\
\hline Control & $3.00(1.66,5.40)^{*}$ & $3.37(1.83,6.21)^{*}$ & $0.41(0.20,0.86)^{*}$ & $0.06(0.03,0.12)^{*}$ \\
\hline \multicolumn{5}{|l|}{ Gender } \\
\hline Female & 1 & 1 & 1 & 1 \\
\hline Male & $1.21(0.68,2.14)$ & $1.17(0.65,2.12)$ & $1.11(0.53,2.32)$ & $0.85(0.47,1.51)$ \\
\hline \multicolumn{5}{|l|}{ Age } \\
\hline$<75$ & 1 & 1 & 1 & 1 \\
\hline$\geq 75$ & $0.46(0.26,0.81)^{*}$ & $0.48(0.27,0.87)^{*}$ & $0.85(0.41,1.77)$ & $1.35(0.76,2.38)$ \\
\hline \multicolumn{5}{|l|}{ Diagnosis } \\
\hline Cancer & 1 & 1 & 1 & 1 \\
\hline Non-cancer & $0.25(0.12,0.54)^{*}$ & $0.54(0.26,1.13)$ & $2.20(0.85,5.66)$ & $1.58(0.78,3.19)$ \\
\hline \multicolumn{5}{|l|}{ Marital status } \\
\hline Single/divorced/widow & 1 & 1 & 1 & 1 \\
\hline Married/defacto & $1.56(0.88,2.76)$ & $1.54(0.86,2.78)$ & $1.16(0.55,2.44)$ & $0.80(0.45,1.41)$ \\
\hline \multicolumn{5}{|l|}{ Aged care facility } \\
\hline Non resident & 1 & 1 & $n / a^{a}$ & 1 \\
\hline Resident & $0.16(0.06,0.44)^{*}$ & $0.15(0.04,0.52)^{*}$ & & $1.85(0.81,4.23)$ \\
\hline \multicolumn{5}{|l|}{$\begin{array}{l}\text { Australian-modified } \\
\text { Karnofsky performance } \\
\text { scale score }\end{array}$} \\
\hline$\leq 40$ & 1 & 1 & 1 & 1 \\
\hline 50 & $1.94(0.85,4.46)$ & $1.33(0.58,3.08)$ & $0.52(0.19,1.43)$ & $0.83(0.36,1.84)$ \\
\hline 60 & $5.36(2.42,11.84)^{*}$ & $1.83(0.86,3.90)$ & $0.19(0.07,0.55)^{*}$ & $0.83(0.40,1.73)$ \\
\hline $70+$ & $3.19(1.31,7.80)^{*}$ & $1.41(0.58,3.44)$ & $0.51(0.18,1.49)$ & $0.71(0.30,1.67)$ \\
\hline \multicolumn{5}{|l|}{ Resource Utilisation } \\
\hline \multicolumn{5}{|l|}{ Group-Activities of } \\
\hline \multicolumn{5}{|l|}{ daily living score } \\
\hline $1-5$ & 1 & 1 & 1 & 1 \\
\hline $6-10$ & $0.60(0.30,1.18)$ & $1.04(0.54,1.99)$ & $0.94(0.39,2.27)$ & $1.56(0.81,3.02)$ \\
\hline $11+$ & $0.05(0.01,0.14)^{*}$ & $0.11(0.04,0.33)^{*}$ & $26.82(5.65,127.46)^{*}$ & $2.13(1.00,4.52)^{*}$ \\
\hline \multicolumn{5}{|l|}{$\begin{array}{l}\text { Inpatient bed-days/100 } \\
\text { days }\end{array}$} \\
\hline$<8$ & $\mathrm{n} / \mathrm{a}$ & $\mathrm{n} / \mathrm{a}$ & 1 & 1 \\
\hline$\geq 8$ & & & $0.11(0.05,0.25)^{*}$ & $0.60(0.34,1.06)$ \\
\hline \multicolumn{5}{|l|}{$\begin{array}{l}\text { Admissions per } 100 \\
\text { days }\end{array}$} \\
\hline$<2$ & $\mathrm{n} / \mathrm{a}$ & $\mathrm{n} / \mathrm{a}$ & 1 & 1 \\
\hline$\geq 2$ & & & $0.15(0.07,0.35)^{*}$ & $0.47(0.26,0.86)^{*}$ \\
\hline \multicolumn{5}{|l|}{ Advance Care Directive } \\
\hline Not completed & 1 & 1 & 1 & $\mathrm{n} / \mathrm{a}$ \\
\hline Completed & $0.60(0.34,1.06)^{*}$ & $0.47(0.26,0.86)^{*}$ & $1.68(0.82,3.46)$ & \\
\hline
\end{tabular}

*Statistically significant at $\leq 0.05$

apatients dying in Residential Aged Care or unknown location omitted from analysis. 
Table 3. Associations between treatment group and 1) Bed-days and 2) number of admissions.

\begin{tabular}{c}
$\geq 8$ Bed-days $\quad \geq 2$ Admissions \\
\hline Odds ratio $_{\text {adj }}$ (95\% confidence interval)
\end{tabular}

\section{Group}

Intervention

Control

$2.09(1.10-3.97)$

$1^{b}$

$p$ value 0.024

$2.67(1.39-5.11)$ 0.003

Adjusted for : ${ }^{a}$ diagnosis(cancer or not), resident of aged care (or not); ${ }^{b}$ age of patient; resident of aged care (or not) 